\title{
Evaluation of the Accuracy and Suitability of Low-Cost RGB-D Sensors for Automated Air Gap Measuring in the Apparel Industry
}

\author{
Yordan K. KYOSEV ${ }^{1}$, Jana SIEGMUND \\ Chair of Assembly Technology for Textile Products, ITM, TU Dresden, Dresden, Germany \\ https://doi.org/10.15221/19.137
}

\begin{abstract}
RGB-Depth motion sensing devices are designed to capture the human body in full size including its motion, whereas gaming versions of these devices are available at acceptable prices for end users. ReconstructMe SDK [1] was already reported as a powerful library which is capable to work with low-cost body scanning devices. Their combination allows the real-time 3D reconstruction of the body and the development of custom applications in $C$ language [2]. Hence, the objective of the current work introduced in this paper is to evaluate the RGB-D sensor of Asus Xtion PRO Live [3] for application in the apparel industry in general and for automated body geometry detection and air gap evaluation in particular. Previous reports on low-cost sensors and systems [4], [5] have demonstrated that these devices already provide sufficient accuracy for certain measurements in the apparel industry. Due to their low price, they could be used in software applications designed for end users. For current evaluations, an upper torso was scanned both without clothing and with shirts of different sizes and colors. Measurement errors were evaluated and an algorithm for automatic gap evaluation based on generated meshes was developed.
\end{abstract}

Keywords: 3D body scanning, low cost, RGB-D sensor, open source, air gap, ReconstructMe SDK

\section{Introduction}

Low-cost hardware devices such as the Asus XTion PRO Live [1] or Microsoft Kinect are already widely used in the gaming industry, and their hardware prices are in an acceptable range for small companies and private customers. Previous reports on low-cost sensors and systems [2], [3] have demonstrated that these devices already provide sufficient accuracy for certain measurements in the apparel industry ; nevertheless, there is still an enormous potential for improvement in terms of measurement accuracy. This work investigates the hardware-based accuracy of an Asus XTion PRO Live sensor and addresses a practical evaluation of its use with ReconstructMe SDK [4], [5].

\section{Hardware-based resolution}

According the technical data of the sensor [1], [6] the image resolution is $640 \times 480$ pixels, whereby color and position coordinates can be obtained for each of them. The area for the measurement is limited to the distance between 0.8 and 3.5 meters from the camera (Fig. 1). Taking into account that the angle for visible objects in the horizontal direction is $58^{\circ}$, the smallest angle $\Delta$, in which the area is recorded at one pixel, is found to be $0.045^{\circ}$ (Eq.1).

$$
\Delta_{\text {horizontal }}=\frac{58^{\circ}}{1280}=0,0453125^{\circ}
$$

If one object is located at a distance $R$ to the camera, the smallest width of the surface cell of this object, which is recorded as one pixel, can be approximatedas

$$
e_{\text {horizontal }} \approx \Delta_{\text {horizontal }} \cdot \frac{\pi}{180} \cdot L
$$

For simplification purposes, the unit cell width is considered to be oriented in straight alignment with the camera.. The unit cell width and object heights depending on the position are calculated and visualized in Figure 2. This diagram demonstrates that at the current depth resolution of $640 \times 480$ (which corresponds to approximately $0.3 \mathrm{MPixel}$ camera!), it is feasible to scan a human with a height of $1.9 \mathrm{~m}$, resulting in a mesh size in the range of $3-4 \mathrm{~mm}$. Thus, the achieved accuracy is already at a sufficient level for the purpose of clothing construction.

\footnotetext{
1 yordan.kyosev@tu-dresden.de ; +49 (0) 351 463-39313; http://tu-dresden.de/mw/itm
} 


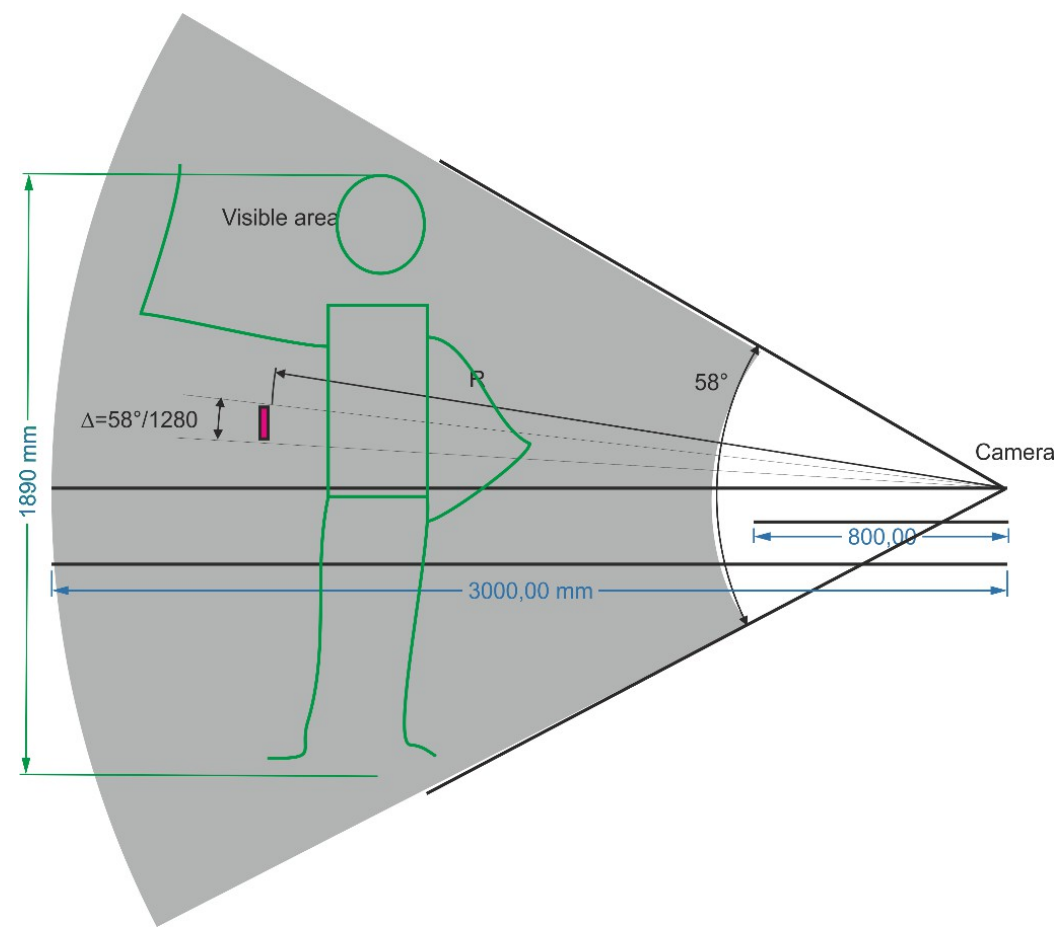

Figure 1. Simplified geometry analysis for the calculation of resolution. The camera angle and depth resolution determine the distance between individual recognizable points forming the surface mesh

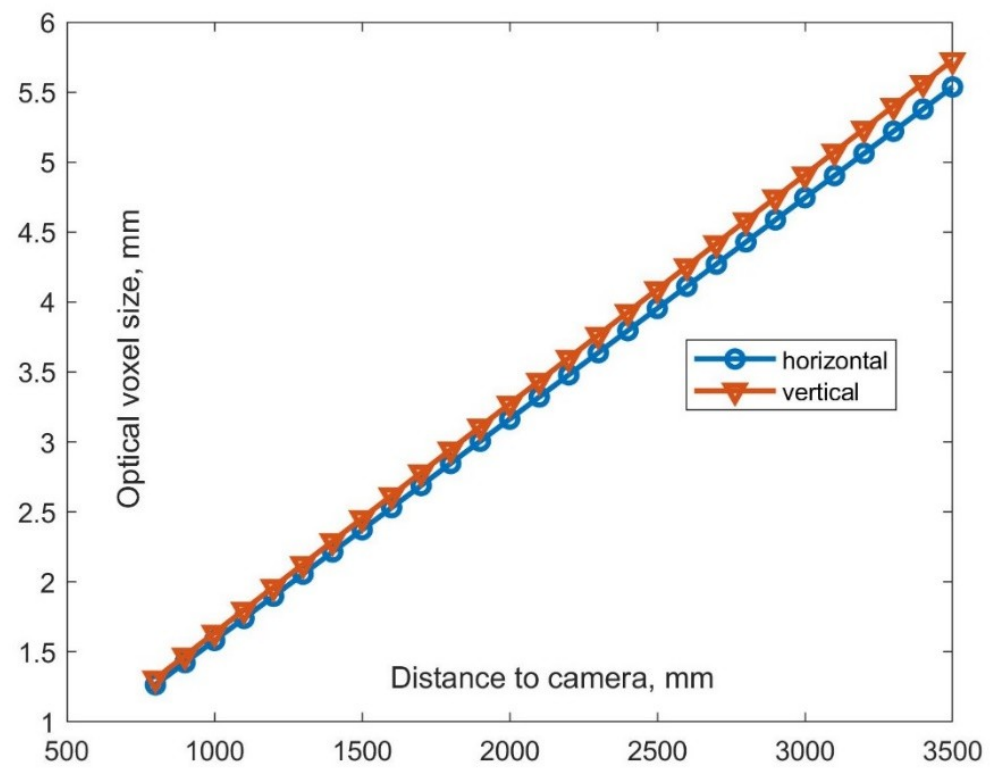

Figure 2. Horizontal and vertical cell sizes of the scanned mesh, depending on the distance to the camera

\section{Practical trials}

For the presented investigations, the upper body of the test person was scanned with and without a T-shirt at a distance of approximately $1 \mathrm{~m}$ from the camera. Scanning was performed based on the standard settings of ReconsructMe SDK [5]. In order to cover the body from all sides, the test person was placed on a rotatable chair, and approximately one rotation per 30 seconds was carried out. The rotational velocity was adjusted by trial and error. At a higher rotational velocity, the software report ost positions and stopped scanning. Figure 3 visualizes the scans - a) without T-shirt, b) with T-shirt. 
As demonstrated in case c), discontinuities occur at the seams; these errors are caused by the three factors inaccuracy of the camera, summation error of the algorithm, and particularly, motion of the test person.

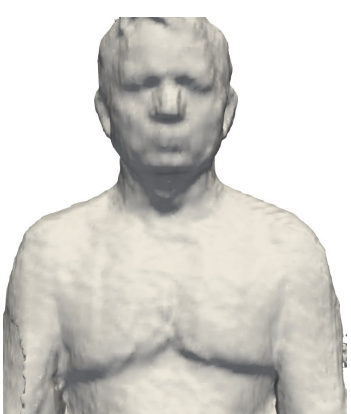

a

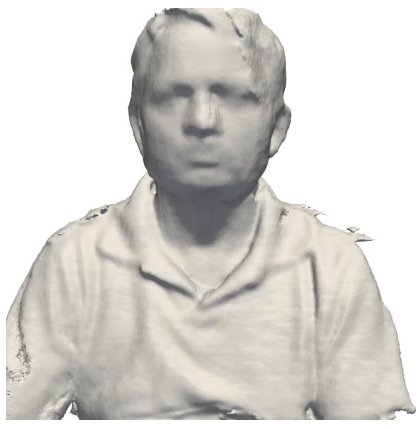

b

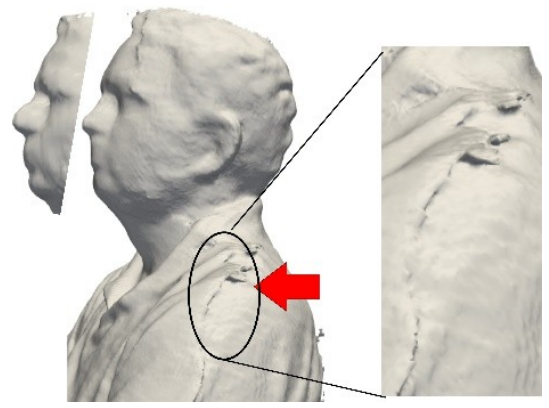

C

Figure 3. Raw data of the scans a) upper torso without shirt, b) with shirt c) side view of $b$ with visible discontinuities of the mesh and different nose form at different scans

\section{Air gap analysis}

For the analysis of air gaps between body and T-shirt both data sets were imported into Geomagic studio [7]. The Geomagic software provides automatic adjustment of the position of both meshes (named "registering"). Figure 4 demonstrates the air gap between cloth and human body at one Z-coordinate in the area of the chest circumference. The real gap between body and cloth cannot be measured accurately in this case; nevertheless, it was observed optically and results correspond with the scanned data. The length of curves resulting from the cross section between body mesh (with and without cloth) and cutting plane presents a possibility for the measurement of body size. These curve lengths can be exported as CSV or DXF file, whereby their length can also be calculated by means of alternative software. For the automatic estimation of body size, special algorithms including a few additional steps are required, where the cutting planes have to be adjusted to be perpendicular to the main body axis if the human is not in a straight vertical position. Additionally, characteristic points of the body have to be defined and recognized. These algorithms are typically implemented in professional body scanner softwares of leading producers.

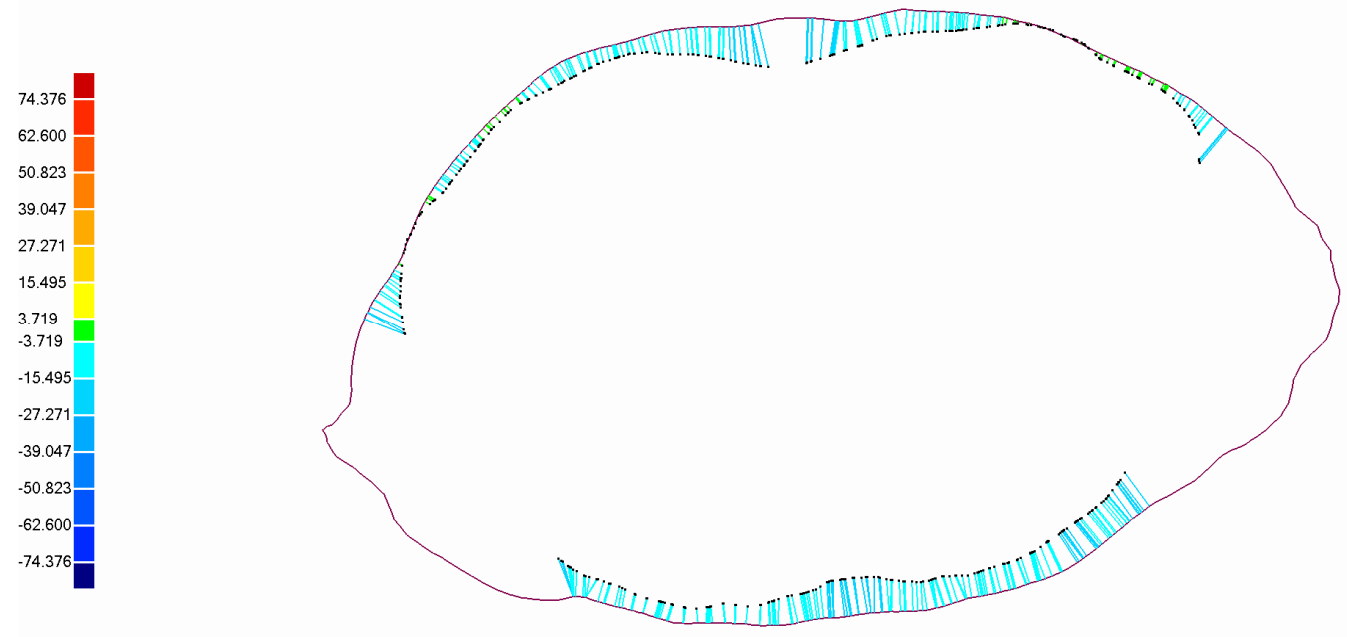

Figure 4. Air gap between T-shirt and human body at one horizontal cut

Figure 5 demonstrates another cross section obtained from the lower part of the scanned body. Here, the small discontinuities that occurred during scanning (region A) as well as within the cloth (region $B$ ) can be observed. These discontinuities are caused by the cloth being flexible and moving during scanning, so it cannot be fixed to remain in the same position. 


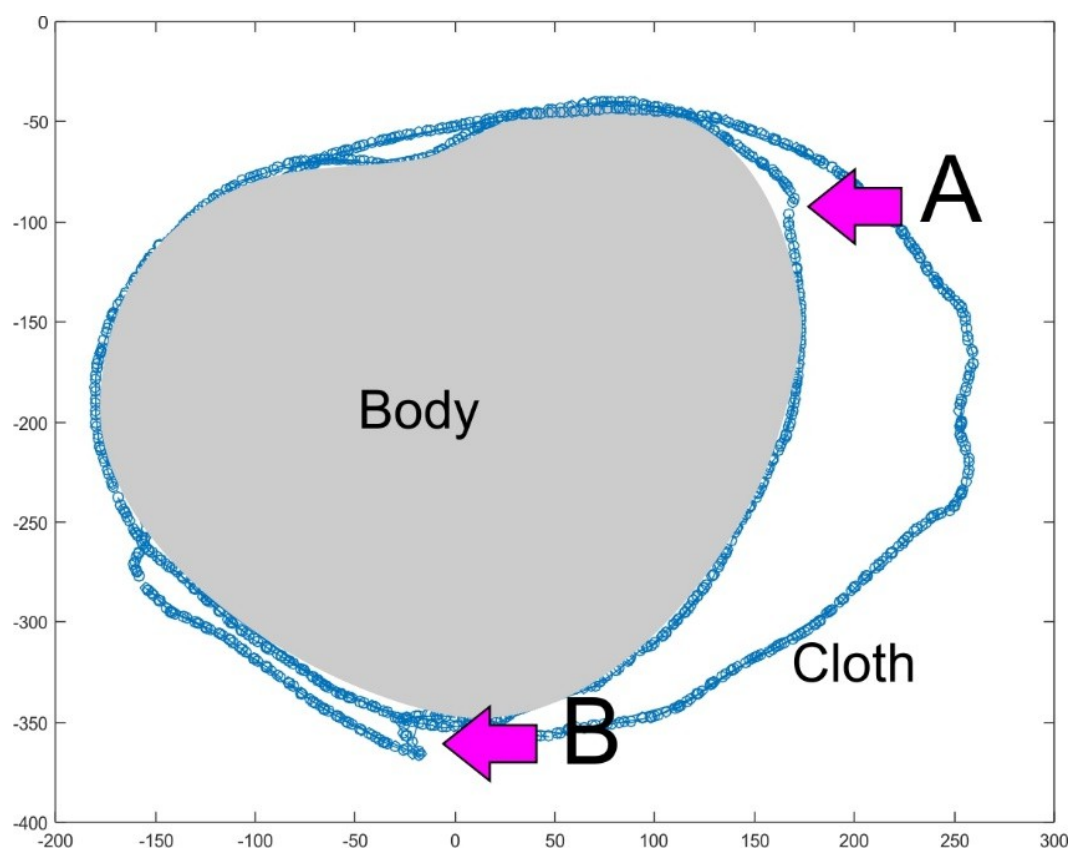

Figure 5. Cross section at another height - with demonstrated larger deviations

Moreover, Figure 6 visualizes the gap size directly at the human body. In the central part of the torso, the presented values are realistic. In contrast, the color indication of the hand areas is not connected with the gap between clothing and human since no cloth was present in this area (short-sleeve shirt). Hence, this figure visualizes that the mesh difference used for gap measurements integrates both the gap between human and textile as well as the changes in human body position in one value. The color indication for the hands reveals the distance between two different positions during scanning.

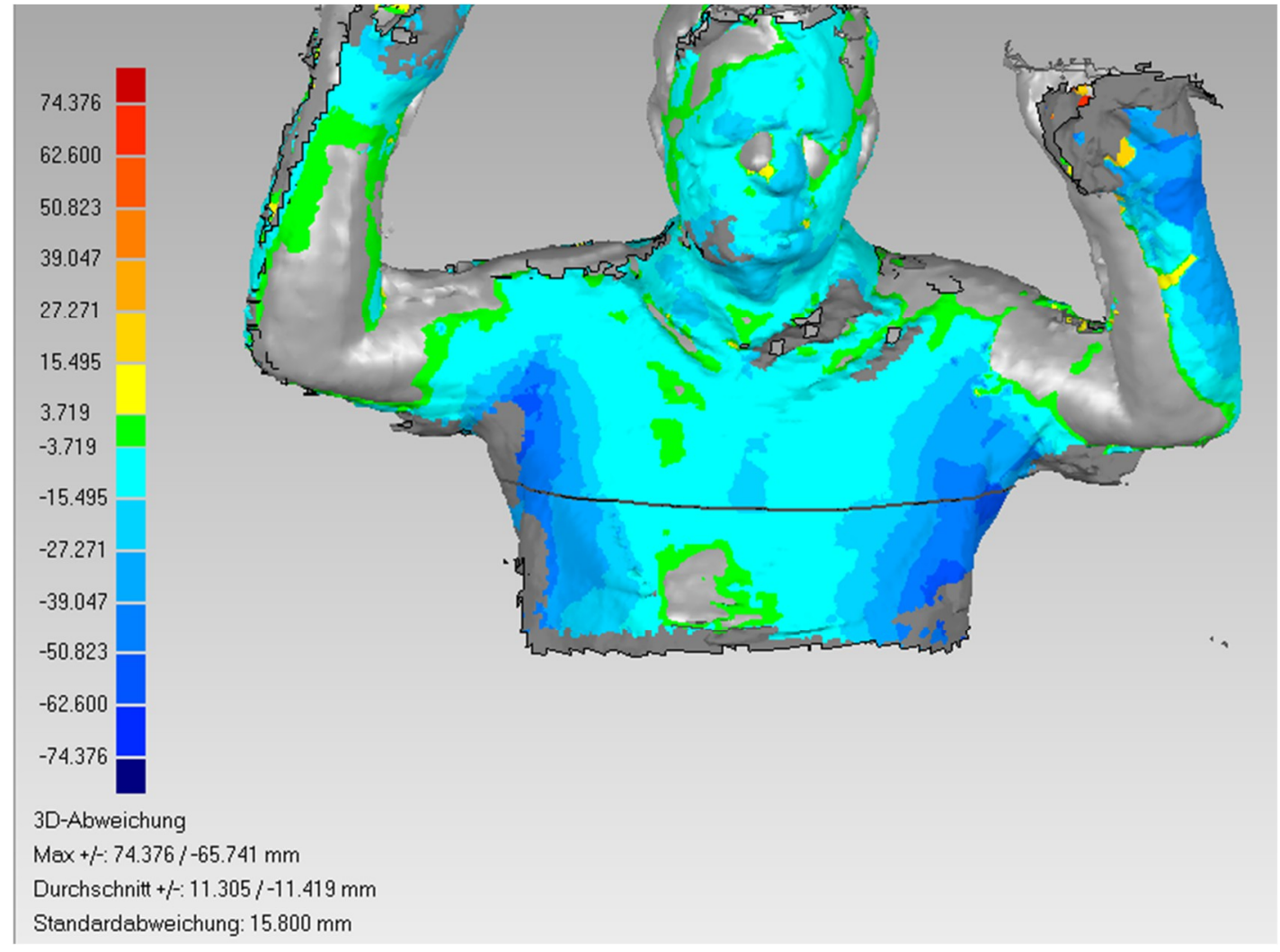

Figure 6. Gap size visualized on the human body 


\section{Conclusions}

The analysis of the hardware accuracy of the Asus RGB-D camera proved a satisfactory level of accuracy for the clothing industry. The mesh size for scanning a complete human body would be in the rage of $3-4 \mathrm{~mm}$ or even better, provided that the body parts to be scanned are located close to the camera. The scanned human upper torso with and without clothing highlighted commonly known problems involved in optical systems and measurements of humans. The camera is capable of ensuring accuracy, yet the motion of the human body leads to discontinuities in the meshes. Additionally, the simple difference between meshes does not provide direct information about the gap between clothing and textile because potential minimal motions of the human body during scanning are also included in the results and are also visualized as differences or gaps. These problems can be overcome if the complete textile product is simulated and considered for the measurement. This method is currently developed and tested at the Chair of Assembly Technology for Textile Products at ITM in Dresden. However, several additional steps and software are still to be developed. The implementation of these steps in an automatic procedure is one of the main challenges for future works.

\section{References}

[1] https://www.asus.com/de/3D-Sensor/Xtion PRO LIVE/

[2] Park, B., Reed, M., A Model-based Approach to Rapid Estimation of Body Shape and Postures Using Low-Cost Depth Cameras, Proceedings of 3DBODY.TECH 2017 Montreal, Canada, 11-12 Oct. 2017, http://dx.doi.org/10.15221/17.281

[3] Weichert, F. et al, Analysis of the accuracy and robustness of the leap motion controller, Sensors 2013, 13, 6380-6393; doi:10.3390/s130506380

[4] Heindl, Ch, et.al, ReconstructMe SDK: a C API for Real-time 3D Scanning, 6th International Conference on 3D Body Scanning Technologies, Lugano, Switzerland, 27-28 October 2015, http://dx.doi.org/10.15221/15.185

[5] http://reconstructme.net/

[6] https://rosindustrial.org/news/2016/1/13/3d-camera-survey

[7] Geomagic https://de.3dsystems.com/software/ 